\title{
Synchronized Babinski and Chaddock Signs Preceded the MRI Findings in a Case of Repetitive Transient Ischemic Attack
}

\author{
Kosuke Matsuzono ${ }^{1,2}$, Takao Yoshiki ${ }^{1}$, Yosuke Wakutani ${ }^{1}$, Yasuhiro Manabe ${ }^{3}$, \\ Toru Yamashita ${ }^{2}$, Kentaro Deguchi $^{2}$, Yoshio Ikeda ${ }^{2}$ and Koji Abe $^{2}$
}

\begin{abstract}
We herein report a 53-year-old female with repeated transient ischemic attack (TIA) symptoms including 13 instances of right hemiparesis that decreased in duration over 4 days. Two separate examinations using diffusion weighted image (DWI) in magnetic resonance imaging (MRI) revealed normal findings, but we observed that both Babinski and Chaddock signs were completely synchronized with her right hemiparesis. We were only able to diagnose this case of early stage TIA using clinical signs. This diagnosis was confirmed 4 days after the onset by the presence of abnormalities on the MRI. DWI-MRI is generally useful when diagnosing TIA, but a neurological examination may be more sensitive, especially in the early stages.
\end{abstract}

Key words: Babinski sign, Chaddock sign, TIA, diffusion weighted image

(Intern Med 52: 2127-2129, 2013)

(DOI: 10.2169/internalmedicine.52.0190)

\section{Introduction}

Transient ischemic attack (TIA) is a clinical syndrome that consists of sudden focal neurologic signs and a complete recovery usually within 24 hours (1). Because TIA can sometimes develop into a cerebral infarction, an early diagnosis is important in avoiding the development of a cerebral infarction (2-4). Magnetic resonance imaging (MRI) is a powerful tool in the diagnosis of TIA, especially when diffusion weighted image (DWI) is performed, as this sequence will reveal abnormalities in $44 \%$ of TIA cases (5-7). However, a negative DWI finding may be misleading during the differential diagnosis for TIA. We herein report a case of repeated transient right hemiparesis (13 instances) with no abnormalities noted on two successive DWI scans, where only the complete synchronization of the Babinski and Chaddock signs with her TIA symptoms allowed us to make the early diagnosis.

\section{Case Report}

A 53-year-old woman suddenly developed right hemiparesis, and she was admitted to our hospital 30 minutes after the onset. She had smoked 10 cigarettes daily for 23 years, and quit at 43 years of age. She used estradiol at a dose of $0.5 \mathrm{mg} /$ day for hormone replacement therapy. Her mother had died from a cerebral infarction at 80 years of age. She had neither hypertension nor diabetes mellitus. A physical examination revealed that she was slightly overweight with a BMI of 27.6. She was alert during the neurological examination, but displayed mild dysarthria as well as a right hemiparesis (Fig. 1A, arrow). Her facial muscles and sensory nerves showed no abnormalities. A motor examination revealed 2/5 strength in her right upper and lower extremities, as outlined by the Medical Research Council of Great Britain (MRC). Her deep tendon reflexes were diminished in her bilateral patellar and achilles due to a lumbar deformation, but Babinski and Chaddock signs were positive on the right side (Fig. 1B, arrow). Hoffmann, Trömner and Warten-

\footnotetext{
${ }^{1}$ Department of Neurology, Kurashiki Heisei Hospital, Japan, ${ }^{2}$ Department of Neurology, Okayama University Graduate School of Medicine, Dentistry and Pharmaceutical Science, Japan and ${ }^{3}$ Department of Neurology, National Hospital Organization Okayama Medical Center, Japan Received for publication January 20, 2013; Accepted for publication May 6, 2013
}

Correspondence to Dr. Koji Abe, kmatsuzono51@gmail.com 

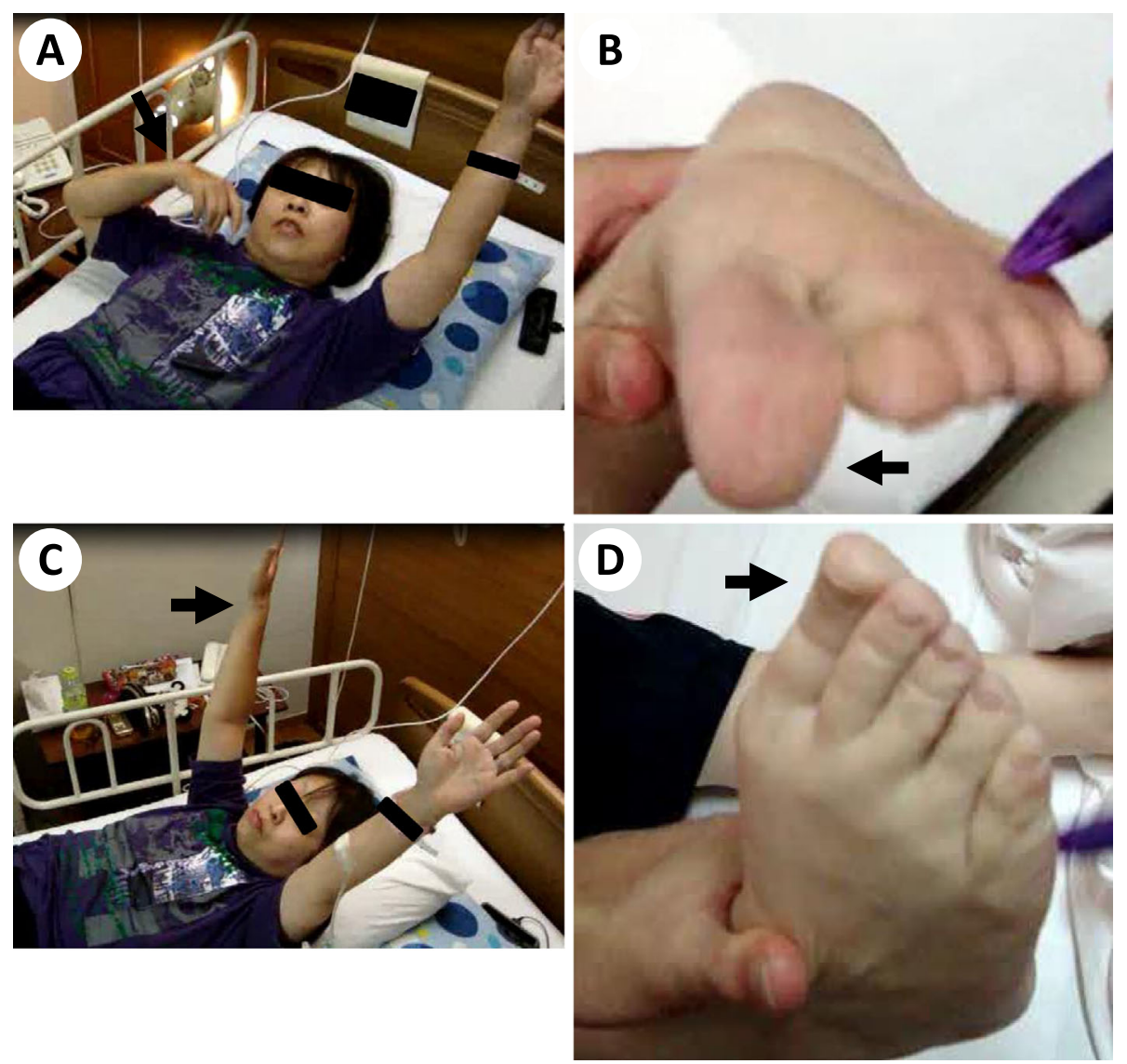

Figure 1. Transient right hemiparesis synchronized with Babinski and Chaddock signs. The appearance of right hemiparesis (A, arrow) corresponded with positive right Babinski signs (B, arrow). The disappearance of right hemiparesis $(C$, arrow) corresponded with the disappearance of right Babinski signs (D, arrow).

berg reflexes were negative bilaterally. Her National Institutes of Health stroke scale (NIHSS) score was 9. A laboratory examination showed a slight polycythemia with a red blood cell count of $532 \times 10^{4} / \mathrm{mL}^{3}$, a white blood cell count of $9,200 / \mathrm{mL}^{3}$ and a platelet cell count of $53.7 \times 10^{4} / \mathrm{mL}^{3}$. Her triglycerids were elevated to $161 \mathrm{mg} / \mathrm{dL}$. Autoantibody tests were negative, including those for antinuclear, rheumatoid factor, anti-Sm, anti-SS-A, anti-SS-B and anti-cardiolipin. Her D-dimer level was $1.16 \mu \mathrm{g} / \mathrm{mL}$, and the results for soluble fibrin monomer complex, $\beta$-thromboglobulin, thrombinantithrombi and, protein $\mathrm{C}$ and $\mathrm{S}$ were normal. A chest $\mathrm{X}$ ray and the ambulatory Holter electroencephalogram readings were normal, and ultrasound echocardiography revealed neither shunt disease nor the presence of thrombi in any cavities. Carotid ultrasonography showed no plaque or stenosis. Although DWI-MRI at 1 hour after the onset was unremarkable, we suspected TIA and administered aspirin at a dose of $100 \mathrm{mg} /$ day as well as edaravone (free radical scavenger). Magnetic resonance angiography (MRA) showed no stenosis or other abnormality.

At 1 hour after the onset, her right hemiparesis disappeared (Fig. 1C, arrow), the moment right Babinski and Chaddock signs became negative (Fig. 1D, arrow) and her NIHSS improved to 0 . However, at 9 hours after the initial onset, right hemiparesis appeared again and right Babinski and Chaddock signs became positive without the appearance of the Hoffmann, Trömner, and Wartenberg reflexes. At this time, DWI-MRI continuted to show normal findings. This second TIA-like phase disappeared 30 minutes later, at the same time that the Babinski and Chaddock signs on the right simultaneously became negative. These attacks repeated for a total of 13 instances with decreasing duration (Fig. 1A-D). When the cranial MRI was performed at 4 days after the onset, high signal intensity at the left corona radiata appeared on DWI; thus, we were able to definitively diagnose the cerebral infarction (Fig. 2). Following standard therapy and rehabilitation, she discharged 18 days after the onset with only slight weakness. She had no additional recurrences of TIA or infarction while being treated with atorvastatin calcium hydrate $(10 \mathrm{mg})$ and aspirin $(100 \mathrm{mg} /$ day $)$.

\section{Discussion}

We herein report a patient with repetitive TIA as indicated by right hemiparesis and Babinski/Chaddock signs. We suspected the cause of the cerebral infarction was hormone replacement therapy because polycythemia improved after the hormone replacement therapy was stopped $(8,9)$. We diag- 


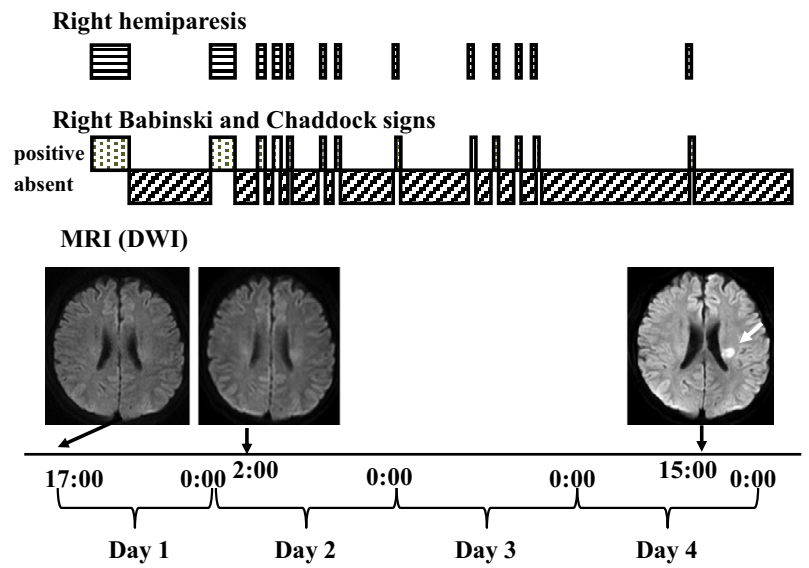

Figure 2. A summary of the clinical course of our case. Babinski and Chaddock signs completely synchronized with the 13 transient right hemiparesis that lasted for $\mathbf{4}$ days after onset. Two successive, early DWI-MRIs showed no abnormalities, but a high signal intensity of the left corona radiata appeared at 4 days after onset (arrow).

nosed this case as a lacunar infarction because arteriosclerosis was not evident and cardiac embolism factors such as paroxysmal atrial fibrillation and patent foramen ovale were not detected. Because there were no initial DWI findings, it was difficult to definitively diagnose her symptoms as TIA. However, the complete synchronization of the Babinski and Chaddock signs with her right hemiparesis led to the early diagnosis of TIA. Previous reports have shown that the duration of the TIA symptoms was significantly longer for DWI-positive patients than for DWI-negative patients; 7.3 hours and 3.2 hours, respectively (5). In our case, the longest duration was about 1 hour, although right hemiparesis occurred a total of 13 times. The short duration of the TIA symptoms may be the reason why two consecutive early MRI-DWI scans could not detect the infarction; alternately, the presence of a perforating artery could also be the cause. A hemodynamic insufficiency of the lenticulostriate artery may have contributed to the exacerbation of the TIA frequency.

Babinski and Chaddock signs are likely related to pyramidal tract lesions, but the complete mechanism has not yet been detected. Synchronized Babinski and Chaddock signs may mean that these signs are the prompt reactions of the corticospinal tract. Because the Hoffmann, Trömner and Wartenberg reflexes did not synchronize in this case, the root of the Babinski and Chaddock signs may be different or may be more prompt reactions than these associated re- flexes.

The DWI-MRI is generally a powerful diagnostic tool for cerebral infarction and TIA, but the present case showed that Babinski and Chaddock signs were more sensitive than DWI in the early phase of this case. In the present case, an early treatment using aspirin and edaravone prevented the development of significant aftereffects. Neurological examinations can detect functional failures under incomplete ischemic processes even when DWI-MRI cannot. Therefore, an early neurological examination may be more sensitive and useful than MRI for diagnosing certain cases of TIA or infarction.

The authors state that they have no Conflict of Interest (COI).

\section{Acknowledgement}

We appreciate the cooperation of the patient. This work was partly supported by a Grant-in-Aid for Scientific Research (B) 21390267 from the Ministry of Education, Science, Culture and Sports of Japan, and by Grants-in-Aid from the Research Committee of CNS Degenerative Diseases (Nakano I), and grants (Mizusawa H, Nishizawa M, Sasaki H, Sobue G) from the Ministry of Health, Labour and Welfare of Japan.

\section{References}

1. Special report from the National Institute of Neurological Disorders and Stroke. Classification of cerebrovascular diseases III. Stroke 21: 637-676, 1990.

2. Dennis M, Bamford J, Sandercock P, Warlow C. Prognosis of transient ischemic attacks in the Oxfordshire Community Stroke Project. Stroke 21: 848-853, 1990.

3. Johnston SC. Clinical practice. Transient ischemic attack. N Engl J Med 347: 1687-1692, 2002.

4. Lovett JK, Dennis MS, Sandercock PA, Bamford J, Warlow CP, Rothwell PM. Very early risk of stroke after a first transient ischemic attack. Stroke 34: e138-e140, 2003.

5. Kidwell CS, Alger JR, Di Salle F, et al. Diffusion MRI in patients with transient ischemic attacks. Stroke 30: 1174-1180, 1990.

6. Winbeck K, Bruckmaier K, Etgen T, von Einsiedel HG, Rottinger M, Sander D. Transient ischemic attack and stroke can be differentiated by analyzing early diffusion-weighted imaging signal intensity changes. Stroke 35: 1095-1099, 2004.

7. Inatomi Y, Kimura K, Yonehara T, Fujioka S, Uchino M. DWI abnormalities and clinical characteristics in TIA patients. Neurology 62: 376-380, 2004.

8. Mueck AO. Postmenopausal hormone replacement therapy and cardiovascular disease: the value of transdermal estradiol and micronized progesterone. Climacteric 15 (Suppl 1): 11-17, 2012.

9. Henderson VW, Lobo RA. Hormone therapy and the risk of stroke: perspectives 10 years after the Women's Health Initiative trials. Climacteric 15: 229-234, 2012.

(C) 2013 The Japanese Society of Internal Medicine http://www.naika.or.jp/imonline/index.html 